\title{
Adopción de comportamientos saludables en la niñez: análisis del concepto
}

\section{RESUMEN}

Objetivo: analizar el concepto de adopción de comportamientos saludables en la niñez. Materiales y método: se utilizó la propuesta de Walker y Avant (2005) para examinar las características definitorias y atributos del concepto. La búsqueda bibliográfica se realizó en las bases de datos Science Direct, PubMed y Scielo, en el período comprendido entre el 2000 y 2016. Resultados: la adopción de comportamientos saludables en la niñez hace referencia al proceso de asimilación o apropiación de conductas, que puede ser ordenado, interrumpido o coordinado, o en algunos casos en el más completo desorden; incluye acciones, actitudes, interacciones y emociones del niño hacia su bienestar, las cuales pueden facilitar, reforzar o mejorar la salud. Constituye un proceso de naturaleza evolutiva, continuamente influenciado por el contexto y puede conducir al establecimiento de un hábito. Conclusiones: la clarificación de este concepto amplia el conocimiento acerca de este fenómeno, lo que permitirá consolidar y plantear estrategias de promoción, prevención e intervención, así como guiar la investigación y la práctica de cuidado de enfermería en la niñez.

\section{PALABRAS CLAVE}

Conductas saludables; niño; salud del niño; promoción de la salud; enfermería (Fuente: DeCS).

Temática: promoción y prevención.

Aporte a la disciplina: el análisis y la clarificación del concepto de adopción de comportamientos saludables en la niñez permitirá su uso efectivo en la investigación de enfermería y su posterior traducción a la práctica, ya que es el primer paso en la comprensión del proceso y los patrones de comportamiento que influyen en el cambio constante de la salud de los niños. Este aporte posibilitará el desarrollo de evidencia para la atención de calidad en la niñez, y facilitará a largo plazo la estructuración de estrategias de promoción desde la perspectiva de los niños.

\section{DOI: 10.5294/aqui.2018.18.2.5}

Para citar este artículo / To reference this article / Para citar este artigo

Moscoso-Loaiza LF, Díaz-Heredia LP. Adopción de comportamientos saludables en la niñez: análisis del concepto. Aquichan 2018; 18(2): 171-185. Doi: 10.5294/aqui.2018.18.2.5

$1 \bowtie$ orcid.org/0000-0002-0580-1865. Universidad Nacional de Colombia, Colombia. Ifmoscosol@unal.edu.co

2 orcid.org/0000-0002-7167-282X. Universidad Nacional de Colombia, Colombia. Ipdiazh@unal.edu.co 


\section{Adoption of Healthy Forms of Behavior During Childhood: Analysis of the Concept}

\section{ABSTRACT}

Objective: Analyze the concept of the adoption of healthy behavior during childhood. Materials and methods: Walker and Avant's proposal (2005) was used to examine the defining characteristics and attributes of the concept. A bibliographic database search was done in Science Direct, PubMed and Scielo for the period between 2000 and 2016. Results: The adoption of healthy behavior during childhood refers to the process whereby forms of behavior are either assimilated or appropriated. It can be an ordered, interrupted, coordinated or, in some cases, completely disordered process. It includes the child's actions, attitudes, interactions and emotions with respect to his or her their well-being, which can facilitate, reinforce or improve health. It is a process of an evolutionary nature that is continually influenced by the context and can lead to the establishment of a habit. Conclusions: Clarification of this concept broadens what we know about the phenomenon. This, in turn, will make it possible to consolidate and propose strategies for promotion, prevention and intervention, as well as to guide research and the practice of nursing care during childhood.

\section{KEYWORDS}

Healthy behavior; child; children's health; health promotion; nursing (Source: DeCS). 


\section{Adoção de comportamentos saudáveís na infância: análise do conceito}

\section{RESUMO}

Objetivo: analisar o conceito de adoção de comportamentos saudáveis na infância. Materiais e método: utilizou-se a proposta de Walker e Avant (2005) para examinar as características definidoras e atributos do conceito. A busca bibliográfica foi realizada nas bases de dados Science Direct, PubMed e SciELO, no período compreendido entre 2000 e 2016. Resultados: a adoção de comportamentos saudáveis na infância faz referência ao processo de assimilação ou apropriação de condutas, que pode ser ordenado, interrompido ou coordenado, ou, em alguns casos, na mais completa desorganização; inclui ações, atitudes, interações e emoções da criança sobre seu bem-estar, as quais podem facilitar, reforçar ou melhorar a saúde. Constitui um processo de natureza evolutiva, continuamente influenciado pelo contexto, e pode levar ao estabelecimento de um hábito. Conclusão: 0 esclarecimento desse conceito amplia o conhecimento acerca do fenômeno, o que permitirá consolidar e propor estratégias de promoção, prevenção e intervenção, bem como orientar a pesquisa e prática de cuidado de enfermagem na infância.

\section{PALAVRAS-CHAVE}

Condutas saudáveis; criança; saúde da criança; promoção da saúde; enfermagem (Fonte: DeCS). 


\section{Introducción}

El comportamiento humano es un factor crucial para la salud (1) debido a la influencia que sobre él ejercen las actitudes, las creencias, los significados y conocimientos, siendo determinantes para las decisiones que las personas toman, y que están presentes en forma de acciones que afectan la salud de manera positiva o negativa (2). La adopción de conductas saludables en la niñez está influenciada por la familia, la escuela, los amigos, los medios de comunicación y la organización social, ya que son aprendidos y, por tanto, susceptibles de ser modificados a lo largo de la vida (3).

La importancia de conocer el fenómeno de la adopción de comportamientos saludables específicamente en los niños entre los 8 y 12 años de edad radica en que esta etapa es descrita como un periodo de cambios neurofisiológicos continuos, en el cual se establece la función ejecutiva que incluye el control de la atención, la memoria de trabajo, el razonamiento, la autorregulación del comportamiento, la inhibición, el procesamiento de información y la fijación de objetivos (4).

Socialmente, esta etapa se caracteriza por el establecimiento de estructuras nuevas que implican el aumento de los niveles de independencia y el mantenimiento de relaciones con los compañeros, la autorregulación, los desafíos intelectuales y los primeros cambios de la pubertad (4). Estos tienen implicaciones para la salud, debido a que si se establecen conductas en este periodo pueden persistir en la transición a la adolescencia y la adultez (5) $\mathrm{y}$, por tanto, mantenerse durante toda la vida.

Es importante reconocer que el niño es un ser activo en su salud, al construir su conducta con base en la interacción con el ambiente y desarrollar patrones de comportamiento que determinarán sus futuras formas de vida (6). Para el desarrollo de este artículo el concepto de "niño" hace referencia a los niños y las niñas entre los 8 y 12 años de edad.

\section{Importancia del análisis del concepto}

Hasta el momento, los resultados de investigación permiten afirmar que el papel de enfermería en el estudio de los comportamientos saludables en los niños se ha fundamentado en sus determinantes, a partir de la visión de los padres, y en las consecuencias que estos tienen sobre la salud del infante, por lo que se han establecido intervenciones que se basan en la alimentación y la actividad física, principalmente (7-13).
La alimentación en la infancia se ha relacionado con la adiposidad y con el riesgo de obesidad. El desarrollo y mantenimiento de un peso excesivo pueden verse afectados por factores ambientales, incluidos los relacionados con la familia, en donde sobresalen los comportamientos de los padres, sus creencias y percepciones, los cuales modelan la ingesta y accesibilidad de los alimentos. Esto implica que la familia es un factor determinante en la adopción de comportamientos saludables (13).

La actividad física en los niños proporciona beneficios para la salud mental y física, pues reduce el riesgo de desarrollar obesidad y enfermedades crónicas a edades tempranas como la diabetes y las enfermedades cardiovasculares. Por el contrario, el tiempo utilizado en conductas sedentarias es determinante de la obesidad infantil $(14,15)$.

La obesidad es el principal factor de riesgo cardiovascular durante la niñez, que contribuye al desarrollo de otros asociados como la hiperglucemia y la hipertensión arterial $(5,9,12,14)$. Por esta razón, la promoción de la salud infantil desde enfermería debe facilitar y potenciar los factores que permiten la adopción de comportamientos que sostengan estilos de vida saludables.

Estos factores deben ser determinados a partir de las respuestas del niño frente a su salud y bienestar, por lo que entender el fenómeno de la adopción de comportamientos saludables a través de la clarificación de su concepto puede permitir la consolidación de estrategias de enfermería efectivas en la promoción y prevención, en donde se vincule de forma articulada a la familia y la escuela, principales actores sociales que enmarcan el desarrollo comportamental del niño.

Fines del análisis. La adopción de comportamientos saludables en la niñez, aunque de gran interés por los efectos que produce a nivel de la salud y el bienestar físico, emocional y social, no se ha definido claramente en la literatura, por lo que tiende a confundirse con otros conceptos, como estilos de vida saludables que, aunque se encuentran vinculados, enmarcan significados diferentes, por lo que el propósito de este análisis es clarificar el significado del concepto de adopción de comportamientos saludables en la niñez, a partir de la construcción de una definición constitutiva.

\section{Materiales y métodos}

Se utilizó la propuesta de Walker y Avant (16), quienes afirman que el análisis de conceptos es una vía para el desarrollo de 
la teoría que proporciona una oportunidad para explicar y describir fenómenos de interés para la práctica, por medio de la cual se examinan las características que definen el concepto y los atributos que permiten decidir qué fenómeno es buen ejemplo y cuál no (16). La búsqueda bibliográfica se realizó en las bases de datos Science Direct, PubMed y SciELO, con los términos de búsqueda "Child", "Health Behavior", "Health Attitude", "Child Behavior" and "Acquisition"; se incluyeron artículos científicos en inglés, español y portugués, en full text, en el periodo comprendido entre el 2000 y el 2016, teniendo en cuenta algunos clásicos de la psicología comportamental, así como capítulos de libros y diccionarios, como se observa en la figura 1. Los artículos científicos fueron seleccionados de forma crítica según los criterios CASPe 2016 (17).

\section{Resultados}

\section{Definiciones y usos del concepto}

- En los descriptores de salud (DeCS) se define el comportamiento como la respuesta observable del ser humano o de un animal ante una situación, y se señala el término conducta como equivalente al comportamiento (19).

- El comportamiento es la manera de conducirse; el conjunto de las acciones con que un ser vivo responde a una situación específica como resultado de la experiencia (20).

Figura 1. Búsqueda bibliográfica para el análisis

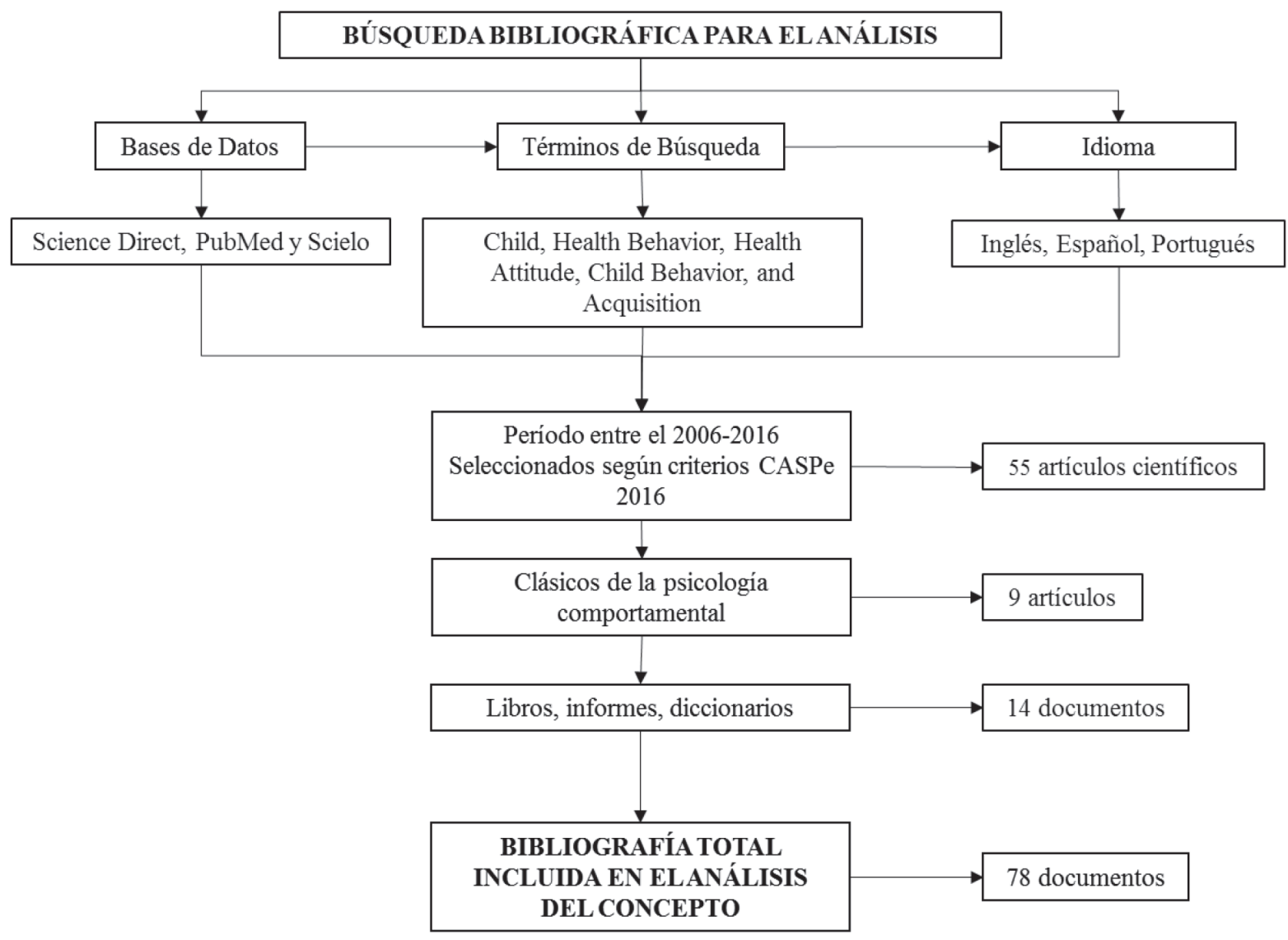

Fuente: Moscoso y Díaz (18). 
- Saludable es la condición armónica del cuerpo, la mente o el alma; implica el tener buena salud y un aspecto sano. Todo lo saludable contribuye a conservar o restablecer la salud (20).

- El comportamiento saludable es la acción llevada a cabo por una persona para mantener, lograr o recuperar la salud, y para prevenir la enfermedad (21).

- La adopción/adquisición hace referencia a elegir o tomar algo como propio, especialmente ideas o costumbres ajenas; hacerse suya una manera de pensar o de actuar, una actitud, una opinión, etc.; así como tomar una resolución o adquirir una determinada configuración (22).

\section{Revisión de la literatura}

A continuación se presentan, en orden cronológico, las diferentes definiciones de conductas saludables y adopción de comportamientos que se encuentran en la revisión de la literatura, para finalizar con una breve descripción de las teorías de adopción y cambio de comportamientos relacionados con la salud que han sido extrapoladas a la investigación infantil, y que permiten establecer los atributos del concepto.

\section{Comportamientos saludables}

- Para Kasl y Cobb (23) los comportamientos saludables son conductas en las que toma parte un individuo cuando goza de buena salud, con el propósito de prevenir la enfermedad.

- McAlister (24) los define como aquellas acciones realizadas por un sujeto que influyen en el bienestar físico, psicológico o social.

- Gochman (25) considera que en los niños se refiere a patrones de comportamiento, acciones y hábitos manifiestos que se relacionan con el mantenimiento de la salud y el bienestar, y con la restauración y mejora de la salud.

- Para Rubio (2) son conductas instrumentales que los individuos realizan y que, disminuyen o aumentan la probabilidad de que se contraiga una enfermedad de forma directa 0 indirecta. Las directas ponen en contacto con el agente patógeno o factor incapacitante (p. e., exponerse a radiaciones solares que devenguen en cáncer de piel). Las indirectas aumentan la vulnerabilidad del organismo, aunque no lo pongan en contacto con tales factores (p. e., una dieta inadecuada).
- En enfermería, los comportamientos saludables hacen referencia a las acciones y actitudes del individuo hacia su salud, las cuales pueden facilitarla y reforzarla, o predisponer el desarrollo de factores de riesgo; están relacionados principalmente con los hábitos dietéticos, la actividad física, el consumo de alcohol y de cigarrillo o tabaquismo (26).

\section{Adopción de comportamientos}

- Bandura (27) considera que pese a que el ambiente físico y social perfila la conducta, las personas no son entes pasivos en este proceso, sino que a su vez pueden influir sobre su propio ambiente, en una dinámica de reciprocidad. Los contextos interpersonales son importantes; el comportamiento, las ideas, los consejos de los demás, y su ayuda o falta de esta, juegan un papel importante en las conductas asociadas a la salud. El círculo de influencia de la persona incluye los seres más cercanos, como familiares, amigos y pares, compañeros de trabajo, profesionales médicos y personas con las que uno se identifique, 0 a las que admire. La dinámica es recíproca: las personas se ven influidas e influyen en los demás.

- Rosenstock, Strecher y Becker (28) consideran que las personas tienen miedo de contraer enfermedades, y los comportamientos asociados a la salud reflejan el nivel de peligro que se percibe, y la reducción del miedo que la persona espera lograr al tomar una acción concreta. Este cálculo incluye la percepción de si el beneficio del cambio de comportamiento supera los obstáculos psicológicos y prácticos, y la decisión de actuar en consecuencia o no.

- Para Prochaska y DiClemente (29), la adopción de un comportamiento nuevo es un proceso, no un acto aislado, ya que las personas tienen diferentes niveles de motivación y voluntad de cambio.

- Rimer y Glanz (30) consideran que las personas atraviesan por una serie de etapas de cambio, por lo que las intervenciones pueden concentrarse en contribuir a la transición de una etapa a la otra, y debido a que puede ser difícil producir cambios dentro de los grupos, se necesita motivación, habilidades nuevas y confianza para llevar a cabo la transición con éxito.

Teorías de adopción y cambio de comportamientos relacionados con la salud del niño

La literatura sobre las actitudes y el comportamiento de la salud de los niños puede organizarse alrededor de dos enfoques. 
El primero fundamentado en la comprensión y el conocimiento de los niños acerca de los conceptos de salud y enfermedad, y el segundo, que las considera desde las diferencias individuales (31). Estos se han desarrollado a través de procesos investigativos con la tendencia inicial de evitar la presencia de niños como participantes activos en los estudios (32), y son con frecuencia los adultos, principalmente padres y profesionales, quienes suministraban las respuestas a las cuestiones que la investigación plantea en relación con los asuntos específicamente infantiles (33-35).

Bajo estos enfoques, las perspectivas teóricas más usadas en el estudio del comportamiento de la salud infantil, por diversas disciplinas como psicología y enfermería, son las siguientes:

- El modelo de creencias de la salud (1950): se centra en el cambio de comportamiento a nivel individual; este modelo identifica cuatro aspectos relevantes: la percepción de la susceptibilidad hacia la gravedad de una enfermedad y sus problemas, los beneficios para reducir el riesgo, y las claves para la acción y la autoeficacia (28). Gholizadeh et al. (36) consideran que el riesgo es percibido como un predictor importante de la mayoría de los comportamientos de salud preventivos, pero que no se aborda en el contexto sociocultural, donde la opinión colectiva y las actitudes pueden influir en los comportamientos.

- Teoría del comportamiento planificado de Icek Ajzen (1988, 1991): explica la relación entre las creencias y los comportamientos. Sustenta que el comportamiento no es completamente voluntario y controlado. Mediante la adición del control percibido conductual aclara la relación entre la intención de la conducta y el comportamiento real (37). Ha guiado la explicación de los comportamientos que se generan en torno a la alimentación de los niños, a partir de las conductas de las madres (38), así como la evaluación del consumo de alcohol en escolares (39).

- Teoría de protección y motivación: desarrollada por Rogers (1975-1983) considera los procesos cognitivos de la evaluación de amenazas y del afrontamiento, en donde los factores que influyen en las intenciones de salud y comportamientos incluyen la percepción de la vulnerabilidad y gravedad al resultado indeseable, la autoeficacia, la eficacia de respuesta y los costos percibidos. Propone que la motivación de la protección se incrementará si la persona advierte la probabilidad y la gravedad de la amenaza para la salud. Sin embargo, no tiene en cuenta el papel de los factores culturales, ambientales y sociales en la adopción o el rechazo de las recomendaciones de salud (40). Se ha utilizado para evaluar los efectos de la promoción de la actividad física (41), y en el establecimiento de factores predictores en el consumo de cigarrillo en adolescentes escolares (42).

- Modelo de Valoración de la Familia de Calgary (1983): valora la estructura, el desarrollo y el funcionamiento familiar, centrándose en los detalles cognitivos, afectivos y conductuales de los individuos que determinan la dinámica familiar (43). Ha sido utilizado para determinar la influencia de los factores ambientales y culturales en el sobrepeso y la obesidad en las familias (44).

- Teoría de la autoeficacia: desarrollada por Bandura como parte de la Teoría social cognitiva (1986). Considera que las medidas de autoeficacia deben ser específicas, contener diversos niveles de dificultad y permitir el registro del grado de la confianza que una persona tiene, acerca de que una conducta determinada será alcanzable. La autoeficacia es un factor importante y constante en la modificación del riesgo y en el cambio de comportamiento (45). Ha sido ampliamente utilizada en la promoción de conductas saludables en las diadas niño/adolescente y sus padres, especialmente en torno a la actividad física y la alimentación (46-49).

- Modelo de promoción de la salud de pender (1982): considera que la conducta está motivada por el deseo de alcanzar el bienestar y el potencial humano. Enfatiza el nexo entre las características personales y las experiencias, conocimientos, creencias y aspectos situacionales vinculados con los comportamientos de salud que se pretenden lograr (50). Tiene en cuenta los beneficios y las barreras percibidos por la acción y la autoeficacia (51). Ha guiado investigaciones para determinar la influencia de las conductas promotoras de salud de los padres en sus hijos (52), así como el desarrollo de herramientas para evaluar los predictores del consumo de alimentos en niños y adolescentes (53).

Estas perspectivas teóricas evidencian la manera como se han explorado la adopción y el cambio de comportamientos de los niños a través del adulto; pese a que estas orientaciones pueden ser de ayuda para motivar el cambio de comportamiento, no toman en cuenta las actitudes y respuestas de los niños frente al ambiente y cómo estas interactúan con el desarrollo cognitivo (31). 


\section{Conceptos relacionados}

La adopción de comportamientos saludables suele estar relacionada en la literatura con los conceptos de estilos de vida y hábitos saludables. Sin embargo, pese a que se vinculan, no son iguales, y difieren cuando se les examina de cerca.

Estilos de vida: son patrones de comportamiento colectivos que se configuran a partir de diferentes elecciones que hacen los seres humanos y están condicionados por las oportunidades de vida que les brinda el contexto en que se desarrollan (54). Incorporan una relación entre las opciones y oportunidades de vida, las cuales se fundamentan en los elementos de la estructura que, junto con los de la agencia, dan forma a los estilos de vida. Los elementos estructurales son las circunstancias de clase, las colectividades, la edad, el género, la etnia y las condiciones de vida, los cuales generan las oportunidades. La agencia, en tanto se trata de las opciones, está condicionada por la socialización y la experiencia de los individuos (55).

Los estilos de vida están determinados por la presencia de factores de riesgo o de factores protectores para el bienestar, por lo cual deben ser vistos como un proceso dinámico que no solo se compone de acciones y comportamientos individuales, sino también de los de naturaleza social (56).

Hábitos saludables: son un set de disposiciones durables para actuar de maneras específicas (57). El hábito es una forma de conservación del pasado, que constituye la habilidad para conducirse de determinada forma, por lo que requiere de aprendizaje y repetición de actos individuales. Un hábito adquirido es, desde el punto de vista fisiológico, una nueva vía de descarga que se forma en el cerebro, por la cual han de escapar ciertas corrientes aferentes (58). Son rutinas o patrones de comportamiento que se establecen en el organismo y que llegan a tener una base orgánica, biológica. La mayoría de estos se vuelven inconscientes, en el sentido en que operan mecánicamente.

Los hábitos se forman desde la infancia temprana, a partir de la existencia de un condicionamiento que sustituye la causa primitiva del acto, el cual se vuelve autónomo cuando se libera cada vez más de los estímulos exteriores y contiene las percepciones reguladoras necesarias. El aprendizaje del hábito se da por medio del adiestramiento. Cada adquisición se refuerza mediante la repetición, la cual facilita el logro de los primeros y el aprendizaje de los siguientes (58).
El habitus constituye la dimensión cultural del hábito, y se entiende como un sistema de esquemas adquiridos, permanentes y transferibles, a partir de los cuales los sujetos perciben el mundo y actúan en él (58). Estos esquemas están estructurados socialmente e interiorizados por medio de la mediación semiótica, se han conformado a lo largo de la historia de cada sujeto y suponen la interiorización de la estructura social, del campo concreto de relaciones sociales en el que el agente se construye como tal (58).

\section{Atributos del concepto}

- Proceso multidimensional: individual y social $(24,29,31$, 32, 39-47).

- Implica múltiples acciones e interacciones que evolucionan y se desarrollan en el tiempo (26-29).

- Está determinado por las características individuales del niño, como son el nivel de conocimiento, las habilidades, las percepciones, las creencias, los valores, las motivaciones, las intenciones para la acción y la percepción de su capacidad para lograr un objetivo $(29,30,31,37-44,48,51)$.

- Tiene influencia del contexto interpersonal, social y organizacional del niño $(21,29,45,46)$.

- Es un proceso recíproco entre el niño y el contexto o ambiente: el niño influye en el contexto y este en el niño, de forma simultánea $(21,29,45,46)$.

- $\quad$ Conduce al bienestar y la salud $(22,25-28,30)$.

\section{Definición del concepto de adopción de comportamientos saludables en la niñez}

La adopción de comportamientos saludables en la niñez hace referencia al proceso de asimilación, aceptación o apropiación de conductas, que puede ser ordenado, interrumpido o coordinado, 0 en algunos casos darse en el más completo desorden; incluye acciones, actitudes, interacciones y emociones del niño hacia su bienestar, las cuales pueden facilitar, reforzar o mejorar la salud. Constituye un proceso de naturaleza evolutiva con diferentes formas, ritmos y pasos, el cual está continuamente influenciado por el contexto, y puede conducir al establecimiento de un hábito.

A continuación se presentan una serie de casos que ilustran el concepto. 


\section{Caso modelo}

Juan es un niño de 8 años de edad que asiste a la escuela y se encuentra en tercer grado. Refiere que le gustan mucho las frutas, especialmente la manzana y el banano, y que las prefiere comer en horas de la tarde después del almuerzo; no consume gaseosas, y solo de vez en cuando consume comida chatarra, ya que su mamá le dice que no es buena para su corazón. Juan sabe que comer frutas y vegetales le ayuda a tener una buena salud. Le gusta jugar fútbol desde los 4 años, ya que su papá le ha enseñado que hacer deporte le ayuda a estar en forma; juega en el parque del conjunto residencial donde vive y en las horas de recreo del colegio, este es su pasatiempo favorito. Juan cuenta que quiere seguir jugando fútbol cuando sea más grande porque se divierte mucho y porque quiere llegar a ser un buen arquero. Sabe que es importante alimentarse bien, ya que si no lo hace, no va a tener la energía suficiente para jugar e ir al colegio. Los padres de Juan señalan que es un niño muy activo y feliz, que es respetuoso, amable y solidario con los otros niños, y que se desempeña bien en las actividades del colegio.

\section{Caso límite}

Juliana es una niña de 10 años de edad que se encuentra en cuarto grado de la escuela primaria. Juliana manifiesta que come algunas frutas en las horas de descanso porque su mamá se las envía en la lonchera, pero cada vez que tiene oportunidad las cambia por paquetes como papas o chitos debido a que estos le parecen más ricos. Juliana no tiene preferencia por ninguna actividad física o deporte en especial, y solo se ejercita en las clases de educación física porque "le toca" y no quiere perder la asignatura. Los padres de Juliana sienten que ella se alimenta bien, y que realiza suficiente actividad física para su edad, además refieren que es una niña responsable y que se desenvuelve bien en todas las actividades del colegio.

\section{Caso contrario}

María es una niña de 11 años de edad, que cursa quinto grado. Su comida preferida es la pizza, junto con los dulces y las gaseosas; refiere que no le gustan los vegetales, pero que las frutas se las come si van acompañadas de leche condensada o helado. María no realiza ninguna actividad física, ya que manifiesta que le da pereza, por lo que prefiere ver televisión durante casi todo el día. En las horas de recreo escoge sentarse a jugar con su celular 0 a chatear con algunas amigas, ya que no le gusta sudar, ni entrar a clase acalorada. Los padres de María se sienten preocupados por su bajo ánimo, por su poca energía para hacer las labores del colegio, y por sus bajas notas. Sin embargo, manifiestan que se sienten tranquilos sabiendo que pasa la mayoría del día dentro de casa, y no la dejan salir al parque porque piensan que es peligroso.

\section{Antecedentes y consecuencias del concepto de adopción de comportamientos saludables en la niñez}

La revisión de la literatura científica nos muestra que la influencia de los padres es uno de los principales determinantes que favorecen la adquisición y el establecimiento de comportamientos saludables en la niñez, mientras que las consecuencias de la falta de estos conlleva el desarrollo de enfermedades crónicas no transmisibles a edades tempranas, como se muestra y amplía en la tabla 1.

Tabla 1. Antecedentes y consecuencias del concepto de adopción de comportamientos saludables en la niñez

\begin{tabular}{|l|}
\hline \multicolumn{1}{|c|}{ Antecedentes del concepto } \\
Influencia de los padres: controlan la disponibilidad y accesibi- \\
lidad de alimentos (restricción, presión para comer, promesa \\
de recompensas) (11, 12), y las posibilidades de práctica de \\
actividad física (AF) en los niños $(10,59)$, desde:
\end{tabular}

a) Estilo de crianza: con resultados diversos

Crianza permisiva (sin restricciones): niños más activos físicamente (60) (61), con mayor riesgo de sobrepeso (62). Crianza autoritaria (respeto por la opinión de los hijos, con límites claros): menos restricciones sobre la dieta $(59,63)$; niños menos propensos a tener sobrepeso (64), y con mayores niveles de $\operatorname{AF}(10,65)$.

Crianza muy autoritaria (muy estricta/disciplinada): niños con mayor riesgo de sobrepeso (61).

\section{b) Nivel educativo de los padres}

Mayor nivel educativo (técnico-universitario): crianza autoritaria. Mayor control sobre la alimentación (59).

Menor nivel educativo (primaria-bachillerato): crianzas permisivas. No control sobre la alimentación (59). 


\section{c) Padres con menor ingreso económico:}

Niños más propensos a pasar mayor tiempo frente a la televisión (66).

Televisión como estrategia para asegurar la estancia de los niños en casa y mantenerlos ocupados en el hogar (66).

Apoyo y aliento de los padres hacia la AF: aumenta la percepción de que los niños participan más en actividades deportivas $(14,15)$, existe más control sobre la televisión y el uso de videojuegos (15).

Las conductas de salud en edades tempranas se asocian con las de edades posteriores $(5,65)$.

\section{Consecuencias del concepto}

La falta de comportamientos saludables en la niñez lleva a:

- Desarrollo de obesidad infantil $(9,12,67,68)$.

- Trastornos del sueño, problemas osteomusculares y respiratorios en los niños $(9,68)$.

- Hipertensión arterial, hiperglucemia, hipertrigliceridemia y elevación de las transaminasas $(12,67,69,70)$.

- Sedentarismo asociado al sobrepeso y obesidad. Aumento del riesgo de adquirir una enfermedad cardiovascular en edades tempranas $(9,14)$.

- Depresión, ansiedad y baja autoestima (71).

- Los factores de riesgo cardiovascular coexisten en la niñez (entre 3 a 5 años) y se refuerzan mutuamente $(67,68,70)$.

Fuente: Moscoso y Díaz (18).

\section{Referentes empíricos}

Para realizar una medición objetiva de la adopción de comportamientos saludables en la niñez se han utilizado principalmente los instrumentos y cuestionarios desarrollados bajo los enfoques teóricos que procuran explicar cómo las actitudes y percepciones que se tengan frente a la propia salud influyen en las conductas saludables de los niños. Estos instrumentos se han utilizado principalmente en los adolescentes y niños mayores de 10 años, y se amplían en la tabla 2.
Tabla 2. Referentes empíricos del concepto de adopción de comportamientos saludables en la niñez

\begin{tabular}{|c|c|}
\hline Instrumento & Descripción \\
\hline $\begin{array}{c}\text { Escala del } \\
\text { Modelo de } \\
\text { Creencias en } \\
\text { Salud diseñada } \\
\text { por Becker en } \\
1974\end{array}$ & $\begin{array}{l}\text { Incluye } 31 \text { ítems que recogen información sobre las di- } \\
\text { mensiones de susceptibilidad a la enfermedad, severi- } \\
\text { dad de la misma, barreras y beneficios percibidos. Este } \\
\text { instrumento se califica a través de una escala Likert con } \\
5 \text { opciones de respuesta, las cuales oscilan entre "to- } \\
\text { talmente en desacuerdo" ( } 1 \text { ) y "totalmente de acuerdo" } \\
\text { (5). De estos reactivos se diseñaron } 12 \text { para evaluar la } \\
\text { severidad, } 6 \text { para la susceptibilidad, } 5 \text { para los beneficios } \\
\text { y } 8 \text { para las barreras, de acuerdo con el análisis factorial } \\
\text { realizado por los autores ( } 72 \text { ). } \\
\text { La confiabilidad interna reportada en un estudio de pro- } \\
\text { moción de la salud con } 80 \text { adolescentes con diabetes } \\
\text { mellitus es de }>0,73 \text {, siendo el valor para cada uno de } \\
\text { los componentes por separado como sigue: susceptibili- } \\
\text { dad }=0,78 \text {, severidad }=0,76 \text {, barreras }=0,76 \text { y benefi- } \\
\text { cios }=0,6(73) \text {. }\end{array}$ \\
\hline $\begin{array}{c}\text { Cuestionario } \\
\text { de la Teoría } \\
\text { de la Conducta } \\
\text { Planeada } \\
\text { desarrollado } \\
\text { por González, } \\
\text { Neipp, Quiles y } \\
\text { Rodríguez-Marín } \\
\text { en el } 2012\end{array}$ & $\begin{array}{l}\text { Compuesto por un total de } 20 \text { ítems agrupados en cua- } \\
\text { tro factores: } 1 \text { ) norma subjetiva, } 4 \text { ítems; } 2 \text { ) intención, } 4 \\
\text { ítems; } 3 \text { ) control comportamental percibido, } 5 \text { ítems; } 4 \text { ) } \\
\text { actitud, } 7 \text { ítems. Con una escala Likert que oscila entre } \\
1 \text { para la actitud más negativa y } 7 \text { para la actitud más } \\
\text { positiva }(74,75) \text {. } \\
\text { Los valores obtenidos con estudiantes entre los } 12 \text { y } 16 \\
\text { años fueron de } 0,85 \text { para el factor control comportamen- } \\
\text { tal percibido, } 0,87 \text { para el factor intención, } 0,76 \text { para el } \\
\text { factor norma subjetiva, y } 0,86 \text { para el factor actitud (76). }\end{array}$ \\
\hline $\begin{array}{l}\text { La Escala de } \\
\text { Autoeficacia } \\
\text { Desarrollada por } \\
\text { Schwarzer en } \\
\text { 1979. Traducida al } \\
\text { español en } 1993\end{array}$ & $\begin{array}{l}\text { Esta escala consta de } 10 \text { ítems construidos a manera de } \\
\text { una escala Likert con } 6 \text { opciones de respuesta, que van de } \\
\text { "totalmente en desacuerdo" (1) a "totalmente de acuer- } \\
\text { do" (6), por lo que la sumatoria del puntaje oscila entre } 6 \\
\text { y } 60 \text { puntos. La versión al español se tradujo por expertos } \\
\text { en } 1993 \text { desde la escala original, con adaptaciones cultu- } \\
\text { rales necesarias para medir de manera contextualizada } \\
\text { el constructo autoeficacia percibida en población costa- } \\
\text { rricense, española y peruana (77, 78). } \\
\text { Los estudios de las características psicométricas de la } \\
\text { Escala de autoeficacia en español han demostrado em- } \\
\text { píricamente su confiabilidad y validez convergente y dis- } \\
\text { criminante. La unidimensionalidad y homogeneidad de } \\
\text { las versiones en español, chino y alemán se demostró a } \\
\text { través del análisis de la correlación ítem-ítem total, factor } \\
\text { carga y componentes principales (79). } \\
\text { Por lo que es una escala general se considera con una } \\
\text { buena capacidad predictiva de las conductas de salud } \\
\text { al tratarse como un recurso personal de afrontamiento. } \\
\text { La escala ha mostrado una consistencia interna alfa de } \\
\text { 0,76 a 0,74 (80). }\end{array}$ \\
\hline
\end{tabular}

Fuente: Moscoso y Díaz (18). 


\section{Conclusiones}

La adopción de comportamientos saludables es un fenómeno universal y social que se da a partir de la interacción de un ser humano con otras personas. Constituye una experiencia subjetiva que se desarrolla bajo diversidad de causas, y produce una gran variedad de consecuencias físicas y psicosociales. Así, la adopción de comportamientos saludables en la niñez es un conjunto abstracto de actitudes, interacciones y emociones del niño hacia su bienestar que influirá en su salud de por vida, por lo que clarificar el concepto amplía el conocimiento acerca del fenómeno, y es el primer paso para lograr entender cómo es su dinámica y conocer sus elementos constitutivos, consolidándolo a partir de la investigación y la práctica de enfermería.

Los comportamientos saludables se adquieren a edades tempranas y persisten en la transición hacia la adultez, por lo que la promoción de la salud en la niñez representa un gran reto para enfermería, que debe facilitar y potencializar los factores que determinan las conductas que sostienen los estilos de vida saludables, a partir de estrategias que incluyan programas escolares y comunitarios que promuevan la actividad física y la nutrición saludable para combatir la carga de enfermedades crónicas asociadas a la vida sedentaria y la obesidad.

Los espacios creados por enfermería para el cuidado infantil deben involucrar a las familias, ya que son los principales agentes sociales que interactúan con el niño, con el fin de cruzar el límite de la comprensión (conocimiento) al de la práctica (actitud), para luego fomentar la adquisición de un patrón de comportamientos saludables que se puedan mantener en el tiempo (hábito), de tal forma que los niños alcancen las etapas de la adolescencia y la adultez de forma óptima.

Conflicto de interés: ninguno declarado.

\section{Referencias}

1. Murphy E. La promoción de comportamientos saludables. Washington, D.C.: Population Reference Boreau; 2005 p. 1-36.

2. Rubio V. Modelos psicológicos del comportamiento saludable. México: Universidad Autónoma de México; 2003.

3. Macias AI, Gordillo LG, Camacho EJ. Hábitos alimentarios de niños en edad escolar y el papel de la educación para la salud. Rev Chil Nutr. 2012;39(3):40-3.

4. Nuru-Jeter AM, Sarsour K, Jutte DP, Thomas W. Socioeconomic predictors of health and development in middle childhood: Variations by socioeconomic status measure and race. Issues Compr Pediatr Nurs. 2010;33(2):59-81.

5. Castellano JM, Peñalvo JL, Bansilal S, Fuster V. Promotion of cardiovascular health at three stages of life: Never too soon, never too late. Rev Esp Cardiol Engl. 2014;67(9):731-7.

6. Reed PG, Shearer NBC. Nursing Knowledge and theory Innovation: Advancing the Science of Practice. New York: Springer; 2011.

7. McMurray RG, Berry DC, Schwartz TA, Hall EG, Neal MN, Li S, et al. Relationships of physical activity and sedentary time in obese parent-child dyads: A cross-sectional study. BMC Public Health. 2015 [citado 2017 mar 15];16(1). Disponible en: http://www.biomedcentral.com/1471-2458/16/124

8. Kim HS, Park J, Park K, Lee M-N, Ham OK. Parent involvement intervention in developing weight management skills for both parents and overweight/obese children. Asian Nurs Res. 2016;10(1):11-7.

9. Cordova A, Villa G, Sureda A, Rodriguez-Marroyo JA, Sánchez-Collado MP. Actividad física y factores de riesgo cardiovascular de niños españoles de 11-13 años. Rev Esp Cardiol. 2012;65(7):620-6.

10. Loprinzi PD, Cardinal BJ, Loprinzi KL, Lee H. Parenting Practices as Mediators of Child Physical Activity and Weight Status. Obes Facts. 2012;5(3):420-30.

11. Smith ADAC, Emmett PM, Newby PK, Northstone K. Dietary patterns and changes in body composition in children between 9 and 11 years. Food Nutr Res. 2014;58(1):22769. 
12. Baughcum AE, Gramling K, Eneli I. Severely obese preschoolers in a tertiary care obesity program: Characteristics and management. Clin Pediatr (Phila). 2015;54(4):346-52.

13. Cislak A, Safron M, Pratt M, Gaspar T, Luszczynska A. Family-related predictors of body weight and weight-related behaviours among children and adolescents: A systematic umbrella review. Family predictors of body weight and related behaviours. Child Care Health Dev. 2012;38(3):321-31.

14. Veitch J, Hume C, Salmon J, Crawford D, Ball K. What helps children to be more active and less sedentary? Perceptions of mothers living in disadvantaged neighbourhoods: What helps children to be more active and less sedentary? Child Care Health Dev. 2013;39(1):94-102.

15. Haines J, O’Brien A, McDonald J, Goldman RE, Evans-Schmidt M, Price S, et al. Television viewing and televisions in bedrooms: Perceptions of racial/ethnic minority parents of young children. J Child Fam Stud. 2013;22(6):749-56.

16. Walker LO, Avant KC. Strategies for theory construction in nursing. 4th ed. Upper Saddle River, N.J: Pearson/Prentice Hall; 2005.

17. CASPe - Critical Appraisal Skills Programme Español [citado 2016 nov 8]. Disponible en: http://www.redcaspe.org/

18. Moscoso LF, Díaz LP. Adopción de comportamientos saludables en niños escolarizados de 8 a 12 años de edad. Facultad de Enfermería. Universidad Nacional de Colombia - Sede Bogotá; 2016.

19. Descriptores de Ciencias de la Salud. Biblioteca Virtual en Salud. 2016 [citado 2017 dic 18]. Disponible en: http://decs. bvs.br/cgi-bin/wxis1660.exe/decsserver/

20. Real Academia Española. Diccionario de la lengua Española. 23a Edición. Planeta Publishing Corporation; 2014 [citado 2016 nov 25]. Disponible en: http://www.rae.es/diccionario-de-la-lengua-espanola/la-23a-edicion-2014

21. Minguet JM, Prada R, Marcilla R. Diccionario Enciclopédico Ciencias de la Salud. Barcelona: Monsa-Prayma Ediciones; 2013.

22. Diccionario Manual de lengua Española / Manual Spanish Language Dictionary. Larousse Editorial; 2013.

23. Kasl SV, Cobb S. Health behavior, illness behavior, and sick-role behavior: ii. sick-role behavior. Arch Environ Health Int J. 1966;12(4):531-41.

24. McAlister AL. Social and environmental influences of health behavior. Health Educ Q. 1981;8(1):25-31.

25. Gochman DS. Labels, systems and motives: Some perspectives for future research and programs. Health Educ Q. 1982;9(2-3):263-70.

26. Andrić A, Vuletić S. Community nurse assessment of cardiovascular behavioural risk factors--a qualitative analysis within the CroHort study. Coll Antropol. 2012;36(Suppl 1):27-34.

27. Bandura A. Social foundations of thought and action: A social cognitive theory. Englewood Cliffs, N.J: Prentice-Hall; 1986.

28. Rosenstock IM, Strecher VJ, Becker MH. Social learning theory and the Health Belief Model. Health Educ Q. 1988;15(2):175-83.

29. Prochaska JO, DiClemente CC, Norcross JC. In search of how people change. Applications to addictive behaviors. Am Psychol. 1992;47(9):1102-14.

30. Rimer BK, Glanz K. Theory at a glance: A guide for health promotion practice. National Cancer Institute; 2005: 57. Report 2. Disponible en: http://www.popline.org/node/276257

31. Tinsley BJ. Multiple influences on the acquisition and socialization of children's health attitudes and behavior: An integrative review. Child Dev. 1992;63(5):1043-69.

32. Contreras C, Perez A. Participación invisible: niñez y prácticas participativas emergentes. Rev Latinoam Cienc Soc Niñez Juv. 2011;2(9):811-25.

33. Gómez JM. El grupo focal y el uso de viñetas en la investigación con niños. EMPIRIA Rev Metodol Cienc Soc. 2012;(24):45-66.

34. Vergara A, Peña M, Chávez P, Vergara E. Los niños como sujetos sociales: el aporte de los nuevos estudios sociales de la infancia y el análisis crítico del discurso. Psicoperspectivas. 2015;14(1):56-65. 
35. Gabaldón S. Aspectos éticos de la investigación en niños y adolescentes. CEA/CEIC. Butlletí del Comité de Bioética De Catalunya. 2012;7:1-8.

36. Gholizadeh L, Davidson P, Salamonson Y, Worrall-Carter L. Theoretical considerations in reducing risk for cardiovascular disease: Implications for nursing practice. Theoretical considerations in reducing risk for cardiovascular disease. J Clin Nurs. 2010;19(15-16):2137-45.

37. Montaño D, Kasprzyk D. Theory of reasoned action, theory of planed behavior, and the integrated behavioral model. En: Glanz K, Rimer BK, Viswanath K, editores. Health Behavior: Theory, Research, and Practice. 5 edition. San Francisco, CA: Jossey-Bass; 2015.

38. Aguirre T, Hudson DB, Weber K, Pozehl B, Boeckner L, Wilhelm S. Mexican american mothers' eating and child feeding behaviors. Issues Compr Pediatr Nurs. 2012;35(1):4-23.

39. Espejo Tort B, Cortés Tomás MT, Gimenéz Costa JA, Luque LE, Ángel R. Elaboración de un cuestionario basado en la Teoría de la Conducta Planificada de Ajzen para evaluar el consumo de alcohol en atracón en adolescentes. Rev Esp Drogodepend. 2011;36(4):403-14.

40. Prentice-Dunn S, Rogers RW. Protection motivation theory and preventive health: Beyond the health belief model. Health Educ Res. 1986;1(3):153-61.

41. Knox ECL, Esliger DW, Biddle SJH, Sherar LB. Lack of knowledge of physical activity guidelines: Can physical activity promotion campaigns do better? BMJ Open. 2013;3(12):e003633.

42. Yan Y, Jacques-Tiura AJ, Chen X, Xie N, Chen J, Yang N, et al. Application of the protection motivation theory in predicting cigarette smoking among adolescents in China. Addict Behav. 2014;39(1):181-8.

43. Cavalcante A, Moreira A, Mendes de Paiva G, Mourão J, Frota N. Aplicação do modelo Calgary para avaliação familiar na estratégia saúde da família. Enferm Bras. 2017;16(2):105-14.

44. Gutiérrez JM, Guevara MC, Enríquez MC, Paz M de los Á, Hernández del Ángel MA, Landeros EA. Estudio en familias: factores ambientales y culturales asociados al sobrepeso y obesidad. Enferm Glob. 2016;16(1):1.

45. Schwarzer R, editor. Self-efficacy: thought control of action. 3 edición. Washington: Hemisphere Pub. Corp; 2014.

46. Woodruff SJ, Kirby AR. The associations among family meal frequency, food preparation frequency, self-efficacy for cooking, and food preparation techniques in children and adolescents. J Nutr Educ Behav. 2013;45(4):296-303.

47. Rutkowski EM, Connelly CD. Self-efficacy and physical activity in adolescent and parent dyads: Self-efficacy and physical activity in adolescent and parent dyads. J Spec Pediatr Nurs. 2012;17(1):51-60.

48. Burke S, Vanderloo L, Anca G, Pearson E, Tucker P. An examination of self-reported physical activity and physical activity self-efficacy among children with obesity: Findings from the Children's Health and Activity Modification Program (CHAMP) Pilot Study. Retos Nuevas Tend Educ Física Deporte Recreación. 2015;28(1):212-8.

49. Kyle T, Hernández A, Reigal R, Morales V. Efectos de la actividad física en el autoconcepto y la autoeficacia en preadolescentes. Retos Nuevas Tend Educ Física Deporte Recreación. 2016;29(1):61-5.

50. Kemppainen V, Tossavainen K, Turunen H. Nurses' roles in health promotion practice: An integrative review. Health Promot Int. 2013;28(4):490-501.

51. Pender NJ, Murdaugh CL, Parsons MA. Health promotion in nursing practice. 6th edition. Upper Saddle River, N.J: Pearson; 2011.

52. Gaete J, Olivares E, Rojas-Barahona CA, Labbé N, Rengifo M, Silva M, et al. Factores asociados a conductas promotoras de salud en adolescentes chilenos. Rev Médica Chile. 142(4):418-27.

53. Dehdari T, Rahimi T, Aryaeian N, Gohari MR, Esfeh JM. Developing and testing a measurement tool for assessing predictors of breakfast consumption based on a health promotion model. J Nutr Educ Behav. 2014;46(4):250-8.

54. Menéndez EL. De sujetos, saberes y estructuras: introducción al enfoque relacional en el estudio de la salud colectiva. Buenos Aires: Lugar Ed; 2010. 
55. Cockerham WC. Medical sociology. En: Cockerham WC, Dingwall R, Quah S, editores. The Wiley Blackwell Encyclopedia of Health, Illness, Behavior, and Society. Chichester, UK: John Wiley \& Sons. 2014 [citado 2017 mar 16]: 1-19. Disponible en: http://doi.wiley.com/10.1002/9781118410868.wbehibs548

56. Álvarez L. Los estilos de vida en salud: del individuo al contexto. Rev Fac Nac Salud Pública. 2012;30(1):95-101.

57. Téllez Iregui G. Pierre Bourdieu: conceptos básicos y construcción socioeducativa. Claves para su lectura. Bogotá: Universidad Pedagógica Nacional; 2002.

58. James W. El hábito. En: Compendio de Psicología. 3a ed. Buenos Aires: Emecé editores; 1963.

59. Philips N, Sioen I, Michels N, Sleddens E, De Henauw S. The influence of parenting style on health related behavior of children: Findings from the ChiBS study. Int J Behav Nutr Phys Act. 2014;11(1):95.

60. Langer SL, Crain AL, Senso MM, Levy RL, Sherwood NE. Predicting child physical activity and screen time: Parental support for physical activity and general parenting styles. J Pediatr Psychol. 2014;39(6):633-42.

61. Jago R, Edwards MJ, Urbanski CR, Sebire SJ. General and specific approaches to media parenting: A systematic review of current measures, associations with screen-viewing, and measurement implications. Child Obes. 2013;9(s1):S-51-S-72.

62. Vollmer RL, Mobley AR. Parenting styles, feeding styles, and their influence on child obesogenic behaviors and body weight. A review. Appetite. 2013;71:232-41.

63. Kakinami L, Barnett TA, Séguin L, Paradis G. Parenting style and obesity risk in children. Prev Med. 2015;75:18-22.

64. Johnson R, Welk G, Saint-Maurice PF, Ihmels M. Parenting styles and home obesogenic environments. Int J Environ Res Public Health. 2012;9(12):1411-26.

65. Lohaus A, Vierhaus M, Ball J. Parenting styles and health-related behavior in childhood and early adolescence: Results of a longitudinal study. J Early Adolesc. 2009;29(4):449-75.

66. Maitland C, Stratton G, Foster S, Braham R, Rosenberg M. The dynamic family home: A qualitative exploration of physical environmental influences on children's sedentary behaviour and physical activity within the home space. Int $\mathrm{J}$ Behav Nutr Phys Act. 2014 [citado 2015 jun 3];11(1). Disponible en: http://www.ijbnpa.org/content/11/1/157

67. Zhou Y, Xie G, Wang J, Yang S. Cardiovascular risk factors significantly correlate with autonomic nervous system activity in children. Can J Cardiol. 2012;28(4):477-82.

68. Imai CM, Gunnarsdottir I, Gudnason V, Aspelund T, Birgisdottir BE, Thorsdottir I, et al. Faster increase in body mass index between ages 8 and 13 is associated with risk factors for cardiovascular morbidity and mortality. Nutr Metab Cardiovasc Dis. 2014;24(7):730-6.

69. Poeta LS, Duarte M de F da S, Caramelli B, Jorge M, Giuliano I de CB. Effects of physical exercises and nutritional guidance on the cardiovascular risk profile of obese children. Rev Assoc Med Bras. 2013;59(1):56-63.

70. Jiménez-Pavón D, Konstabel K, Bergman P, Ahrens W, Pohlabeln H, Hadjigeorgiou C, et al. Physical activity and clustered cardiovascular disease risk factors in young children: A cross-sectional study (the IDEFICS study). BMC Med. 2013 [citado 2017 mar 16];11(1). Disponible en: http://bmcmedicine.biomedcentral.com/articles/10.1186/1741-7015-11-172

71. Saunders TJ, Chaput J-P, Tremblay MS. Sedentary behaviour as an emerging risk factor for cardiometabolic diseases in children and youth. Can J Diabetes. 2014;38(1):53-61.

72. Ortiz G, Ortega É. Capacidad predictiva de la adherencia al tratamiento en los modelos sociocognitivos de creencias en salud. Psicol Salud. 2011;21(1):79-90.

73. Butts JB, Rich K, editores. Philosophies and theories for advanced nursing practice. Sudbury, Mass: Jones and Bartlett Publishers; 2011.

74. Neipp MC, Quiles MJ, León E, Tirado S, Rodríguez-Marín J. Aplicando la Teoría de la Conducta Planeada: ¿qué factores influyen en la realización de ejercicio físico? Aten Primaria. 2015;47(5):287-93.

75. González ST, López MCN, Marcos YQ, Rodríguez-Marín J. Development and validation of the theory of planned behavior questionnaire in physical activity. Span J Psychol. 2012;15(02):801-16. 
76. Huéscar E, Rodríguez-Marín J, Cervelló E, Moreno-Murcia JA. Teoría de la acción planeada y tasa de ejercicio: Un modelo predictivo en estudiantes adolescentes de educación física. An Psicol. 2014 [citado 2017 nov 4];30(2). Disponible en: http://revistas.um.es/analesps/article/view/162331

77. Schwarzer R, editor. Self-efficacy: thought control of action. Washington: Hemisphere; 1992.

78. Cid HP, Orellana YA, Barriga O. Validación de la escala de autoeficacia general en Chile. Rev Médica Chile. 2010 [citado 2017 nov 9];138(5). Disponible en: http://www.scielo.cl/scielo.php?script=sci_arttext\&pid=S0034-98872010000500004\&lng $=$ en\&nrm=iso\&tlng=en

79. Schwarzer R, Bäßler J, Kwiatek P, Schröder K, Zhang JX. The assessment of optimistic self-beliefs: Comparison of the german, spanish, and chinese versions of the General Self-efficacy Scale. Appl Psychol. 1997;46(1):69-88.

80. Carissimi A, Adan A, Tonetti L, Fabbri M, Hidalgo MP, Levandovski R, et al. Physical self-efficacy is associated to body mass index in schoolchildren. J Pediatr (Rio J). 2017;93(1):64-9. 